\title{
High-accuracy Modeling of Ship Deformation Based on Inertial Measuring Method
}

\author{
Er-kai Yuan \\ Beijing University of Aeronautics and Astronautics \\ Beijing, China \\ 93716 Unit of PLA A.F, Tianjin 301700 China \\ e-mail: 642101136@qq.com
}

\author{
Gong-liu Yang \\ School of Instrument Science \& opto-electronics \\ Engineering \\ Beijing University of Aeronautics and Astronautics \\ Beijing, China \\ e-mail: bhu17-yang@139.com
}

\begin{abstract}
High-accuracy modeling is the key problem of ship deformation measurement based on inertial measuring method. This paper is to find a high-accuracy modeling method based on the ship deformation data measured by optical devices. According to different modeling methods, mathematical models are designed by the analysis of the initial measurement data. Wavelet analysis method is introduced in the process of modeling. Attitude matching method is selected as the simulation algorithm and Kalman filter equations are set up based on the algorithm. Simulation results show that the ship deformation can be represented accurately by mathematical models. The Precision Estimation result is better than 0.4".
\end{abstract}

Keywords: high-accuracy modeling; ship deformation measurement; inertial measuring method; Wavelet analysis; Kalman filter

\section{INTRODUCTION}

Nowadays naval vessels are equipped with a large number of attack and defense devices such as radar antennas, launching gears, optical systems, etc. In order to keep them working in a high degree of accuracy, accurate attitude and motion parameters are required in practice. Owing to the ship deformation, attitude errors are engendering between the peripheral apparatus and the central devices, which directly affect the accuracy of the devices. The problems of evaluation and proper allowance for ship deformation become particularly urgent for large-sized ships [1].

Among various methods for measuring ship deformation, optical, photographic and photogrammetric methods are the most widespread. But often they are not universal or accurate enough. The measuring method based on inertial elements matching is becoming the most popular method in this field nowadays. This method expects to estimate the ship deformation with a mathematical algorithm. So a key point of the method is how to establish high-accuracy modeling of ship deformation. The modeling is complicated because many factors result in ship deformation, such as motion disturbances, sea waves, helm's operation, redistribution of freight and fuel on it and non-uniform heating of different parts of the vehicle under the sun [2] [3].

This paper is to verify the accuracy of the modeling methods for ship deformation based on the ship deformation data measured by optical devices. The measurement data's theoretical precision is better than 3 " and it can be regarded as the real ship deformation. Attitude matching method is selected as the simulation algorithm. According to different modeling methods, different mathematical models are designed by the analysis of the initial measurement data. Wavelet analysis method is introduced in the process of modeling. Verification of the models is done by simulation and high-accuracy modeling method of ship deformation is determined.

\section{SHIP DEFORMATION DEFINITION AND ATTITUDE MATCHING METHOD}

\section{A. Ship Deformation Definition}

Coordinate systems definition of measuring method based on inertial measuring method is shown in figure.1. Three-axis laser gyro unit (LGU) is selected as the inertial measuring unit (IMU). The LGUl's axes oxyz are aligned with the coordinate system of the ship, and the axis oy is aligned with the longitudinal axis of the ship, oz is perpendicular to the deck and directed upwards, ox supplements the two previous axes to form the right-handed orthogonal coordinate system. Let us introduce the following designation: $\Phi_{\mathrm{z}}$ as a bend angle in the plane of the deck; $\Phi_{\mathrm{x}^{-}}$ a bend angle in the longitudinal plane of the ship; $\Phi_{y^{-}}$a torsion angle around the longitudinal axis [4].

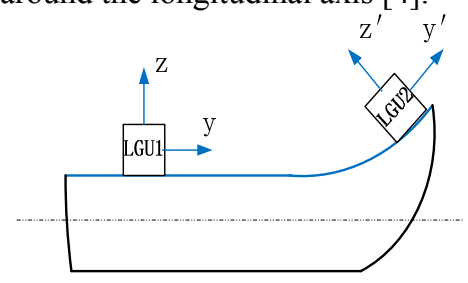

Figure 1. Ship deformation definition.

\section{B. Attitude Matching Method}

This paper selects attitude matching method as the simulation algorithm (a rather complete review of this method can be found in [5]).

LGU1's carrier coordinate system is $b_{1}$, and LGU2's is $b_{2}$. $i_{1}$ and $i_{2}$ are coordinate systems fixed by $b_{1}$ and $b_{2}$ at the start time $t_{0}$. Using the attitude angle increment outputs of LGU1 and LGU2, attitude transfer matrixes $\mathrm{C}_{b 1}^{i 1}$ and $\mathrm{C}_{b 2}^{i 2}$ update constantly. According to the algorithm, observable $\mathrm{Z}$ based on attitude matching method is determined by $\mathrm{C}_{b 1}^{i 1}$ and $\mathrm{C}_{b 2}^{i 2}$.

$$
Z=B \Phi-A \Phi_{0}
$$


Where $\Phi$ is the ship deformation obtained by calculation and $\Phi_{\mathrm{i}}=\varphi_{\mathrm{i}}+\theta_{\mathrm{i}}, \mathrm{i}=\mathrm{x}, \mathrm{y}, \mathrm{z} . \Phi_{0}$ is the initial deformation at the start time $t_{0}$. $Z$, A and $B$ can be calculated by attitude transfer matrixes $\mathrm{C}_{b 1}^{i 1}$ and $\mathrm{C}_{b 2}^{i 2}$ and the matrix forms are:

$$
\begin{gathered}
\mathrm{C}_{b 1}^{i 1}=\left[C_{i j}\right]_{3 \times 3} \quad \mathrm{C}_{b 2}^{i 2}=\left[C_{i j}^{\prime}\right]_{3 \times 3} \\
A=\left|\begin{array}{lll}
C_{33} C_{22}^{\prime}-C_{23} C_{32}^{\prime} & C_{13} C_{32}^{\prime}-C_{33} C_{12}^{\prime} & C_{23} C_{12}^{\prime}-C_{13} C_{22}^{\prime} \\
C_{33} C_{21}^{\prime}-C_{23} C_{31}^{\prime} & C_{13} C_{31}^{\prime}-C_{33} C_{11}^{\prime} & C_{23} C_{11}^{\prime}-C_{13} C_{21}^{\prime} \\
C_{31} C_{22}^{\prime}-C_{21} C_{32}^{\prime} & C_{11} C_{32}^{\prime}-C_{31} C_{12}^{\prime} & C_{21} C_{12}^{\prime}-C_{11} C_{22}^{\prime}
\end{array}\right| \\
Z=\left|\begin{array}{lcc}
C_{13} C_{12}^{\prime}+C_{23} C_{22}^{\prime}+C_{33} C_{32}^{\prime} \\
C_{13} C_{11}^{\prime}+C_{23} C_{21}^{\prime}+C_{33} C_{31}^{\prime} \\
C_{11} C_{12}^{\prime}+C_{21} C_{22}^{\prime}+C_{31} C_{32}^{\prime}
\end{array}\right| B=\left|\begin{array}{ccc}
1 & 0 & 0 \\
0 & -1 & 0 \\
0 & 0 & -1
\end{array}\right|
\end{gathered}
$$

It's a linear equation about $\Phi$ and $\Phi_{0}$. Optimal estimation of ship deformation can be carried out by the Kalman filter [6] [7].

\section{MODELING METHODS OF SHIP DEFORMATION.}

The ship deformation can be divided into dynamic deformation $\theta$ and static deformation $\varphi$. The separation is based on the change rate of the deformation. The equation of $\Phi, \theta$ and $\varphi$ is: $\Phi=\varphi+\theta$.

Generally speaking, two different modeling methods are used for simulation in pertinent literatures. One method identifies the static deformation to a constant value and using a second-order Markov process to represent the dynamic deformation. All trends of the ship deformation are treated as dynamic deformation. The other method is identifying the static deformation to a slow-varying value and using a second-order Markov process to represent the dynamic deformation [8]. The difference is the definition of static deformation. Different mathematical models are designed as follows by the analysis of the initial measurement data according to the two modeling methods.

\section{A. Modeling Method Considering the Slow Varying of Static Deformation}

This method is to represent the static and dynamic deformation with different mathematical models separately.

The frequency spectrum analysis result of $\Phi_{x}$ is shown in Figure 2. The peak in the high- frequency area (more than $0.4 \mathrm{~Hz}$ ) is the spectrum of high-frequency noise. The ship deformation measurement data carries a large number of high-frequency noises. The noises are mainly caused by equipment vibration and it's not the component of ship deformation. It can be filtered out without affecting the deformation component.

The spectrum curve also shows that the frequency characteristics of dynamic deformation and static deformation are significantly different. This is the basis for the separation of the deformation component.

Wavelet analysis method is introduced in the process of modeling. The ship deformation data is a time series. Wavelet analysis method can do frequency characteristics analysis in detail of the series so it can effectively extract information from the series. The method can be used to filter out high-frequency noises and separate static deformation and dynamic deformation [9].

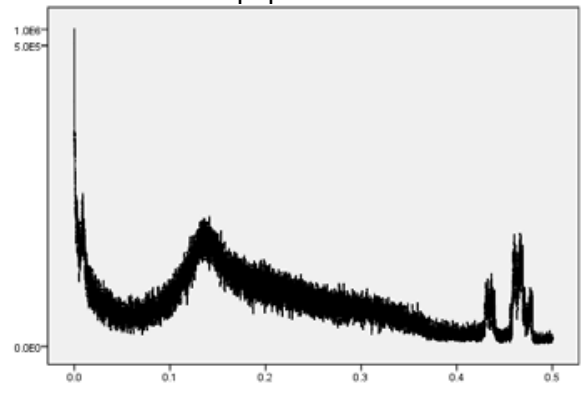

Figure 2. Spectrum curve of $\Phi x$ (x: frequency, y: density).

The separation result shows that static deformation $\varphi$ has no regular pattern, and no proper mathematical model can represent it directly. Considering the static deformation changes very slowly, it's reasonable to analyze and modeling the static deformation angular velocity $\omega_{\varphi}\left(\omega_{\varphi}=\dot{\varphi}\right)$.

Value of $\omega_{\varphi}$ is very small $\left(10^{-5} \mathrm{deg}\right)$, and it always fluctuates around zero. So, $\omega_{\varphi}$ can be regarded as a zeromean random process, which can be represented by correlation function:

$$
\mathrm{K}_{\theta_{\mathrm{i}}}(\tau)=\delta_{\mathrm{i}}^{2} \mathrm{e}^{-\mu_{i}|\tau|} \quad \mathrm{i}=\mathrm{x}, \mathrm{y}, \mathrm{z}
$$

Where $\mu_{\mathrm{i}}$ is the irregularity coefficient, reciprocal value of correlation time; $\delta_{\mathrm{i}}$-the standard deviation (STD) of $\omega_{\varphi}$. Correlation test shows that the correlation time of $\omega_{\varphi}$ is more than 100 s (confidence interval is $60 \%$ ). The process can be approximated as a zero-mean random walk process and the shaping filter equation with such a correlation function will be:

$$
\dot{\omega}_{\varphi_{\mathrm{i}}}=\delta_{\mathrm{i}} \mathrm{w}(\mathrm{t}) \quad \mathrm{i}=\mathrm{x}, \mathrm{y}, \mathrm{z}
$$

$\delta_{\mathrm{i}}$ can be directly calculated as a statistic of $\omega_{\varphi}$.

The dynamic deformation components may be represented as random processes approximated by the correlation function:

$$
\mathrm{K}_{\theta_{\mathrm{i}}}(\tau)=\sigma_{\mathrm{i}}^{2} \mathrm{e}^{-\mu_{i}|\tau|}\left(\cos \lambda_{\mathrm{i}} \tau+\frac{\mu_{i}}{\lambda_{i}} \sin \lambda_{i}|\tau|\right) \mathrm{i}=\mathrm{x}, \mathrm{y}, \mathrm{z}
$$

Where $\mu_{\mathrm{i}}$ is the irregularity coefficient, reciprocal value of correlation time; $\lambda_{i}$ - the prevailing variation frequency of dynamic deformation; $\sigma_{i}$-the STD of dynamic deformation. It's a second-order Markov process and the shaping filter equation with such a correlation function will be:

$$
\ddot{\theta}_{i}+2 \mu_{i} \dot{\theta}_{i}+b_{i}^{2} \theta_{i}=2 b_{i} \sigma_{\mathrm{i}} \sqrt{\mu_{i}} w(t) \quad \mathrm{i}=\mathrm{x}, \mathrm{y}, \mathrm{z}
$$

Where $b_{i}^{2}=\mu_{i}^{2}+\lambda_{i}^{2}, \mathrm{w}(\mathrm{t})$ is the white noise.

The accuracy of modeling depends on the reliability of the parameters, $\mu_{\mathrm{i}}, \lambda_{i}, \sigma_{\mathrm{i}}$. These parameters can be calculated by mathematical statistics of dynamic deformation data.

$\lambda_{i}$ is determined by the frequency characteristics. Spectrum curves can show the prevailing variation frequency of dynamic deformation obviously. Value on $\mathrm{x}$-axis at the 
peak in the middle- frequency area (between $0.1 \mathrm{~Hz}$ and $0.4 \mathrm{~Hz}$ ) is the value $\lambda_{i} \cdot \sigma_{\mathrm{i}}$ can be directly calculated as a statistic of dynamic deformation. Calculation of $\mu_{\mathrm{i}}$ needs correlation test.

Based on algorithm above, the Kalman filter state vector is of the $[1 \times 15]$ size:

$\mathrm{X}=\left|\Phi_{0_{x}}, \Phi_{0 y}, \Phi_{0 z}, \varphi_{x}, \varphi_{y}, \varphi_{z}, \omega_{x}, \omega_{y}, \omega_{z}, \theta_{x}, \theta_{y}, \theta_{z}, \dot{\theta}_{x}, \dot{\theta}_{y}, \dot{\theta}_{z}\right|^{T}$

Definitions of the elements are represented above. The system state equation can be represented in a matrix form as:

$$
\dot{X}=F X+W
$$

$F$ is the system matrix according to the mathematical models designed in the last section and its matrix form is:

$$
\begin{aligned}
& F=\operatorname{diag}\left\{\left[0_{3 \times 3}, F_{6 \times 6}^{\prime}, F_{6 \times 6}^{\prime \prime}\right]\right\} \\
& F_{6 \times 6}^{\prime}=\left[\begin{array}{ll}
0_{3 \times 3} & I_{3 \times 3} \\
0_{3 \times 3} & 0_{3 \times 3}
\end{array}\right] \quad F_{6 \times 6}^{\prime \prime}=\left[\begin{array}{cc}
0_{3 \times 3} & I_{3 \times 3} \\
\mathrm{C} & C^{\prime}
\end{array}\right] \\
& C=\operatorname{diag}\left\{\left[-b_{x}^{2},-b_{y}^{2},-b_{z}^{2}\right]\right\} C^{\prime}=\operatorname{diag}\left\{\left[-2 \mu_{x},-2 \mu_{y},-2 \mu_{z}\right]\right\}
\end{aligned}
$$

$W$ is the system noise matrix and its nonzero elements are:

$$
\mathrm{W}(7)=\delta_{x}, \mathrm{~W}(8)=\delta_{y}, \mathrm{~W}(9)=\delta_{z}
$$

$\mathrm{W}(13)=2 b_{x} \sigma_{x} \sqrt{\mu_{x}}, \mathrm{~W}(14)=2 b_{y} \sigma_{y} \sqrt{\mu_{y}}, \mathrm{~W}(15)=2 b_{z} \sigma_{z} \sqrt{\mu_{z}}$

The measurement equation can be represented as:

$$
Z=H X+V
$$

Due to the equation (1), the measurement matrix is taking the form of:

$$
H=\left[-A, B, 0_{3 \times 3}, B, 0_{3 \times 3}\right]
$$

The definitions of $\mathrm{A}, \mathrm{B}, \mathrm{Z}$ are the same as equation (1).

\section{B. Modeling Method Identifing the Static Deformation to a Constant Value}

This method identifies the static deformation to a constant value. Dynamic deformation is still a second-order Markov process. The model parameters are defined the same as above can be calculated the same way with the initial measurement data filtered out high-frequency noises.

Due to this method, the Kalman filter needs to be changed as follow:

$$
\begin{gathered}
\mathrm{X}=\left|\Phi_{0_{x}}, \Phi_{0 y}, \Phi_{0 z}, \varphi_{x}, \varphi_{y}, \varphi_{z}, \theta_{x}, \theta_{y}, \theta_{z}, \dot{\theta}_{x}, \dot{\theta}_{y}, \dot{\theta}_{z}\right|^{T} \\
F=\operatorname{diag}\left\{\left[0_{6 \times 6}, F_{6 \times 6}^{\prime \prime}\right]\right\}
\end{gathered}
$$

$F_{6 \times 6}^{\prime \prime}$ is defined the same as above. Nonzero elements of $\mathrm{W}$ are:

$$
\mathrm{W}(10)=2 b_{x} \sigma_{x} \sqrt{\mu_{x}}, \mathrm{~W}(11)=2 b_{y} \sigma_{y} \sqrt{\mu_{y}}, \mathrm{~W}(12)=2 b_{z} \sigma_{z} \sqrt{\mu_{z}}
$$

The measurement matrix is $H=\left[-A, B, B, 0_{3 \times 3}\right]$

\section{SIMULATION AND ANALYSIS}

\section{A. Simulation conditions}

This paper is aiming at verifying the accuracy of the modeling methods. So the simulation will not bring in extra errors, such as erection error, gyro drift error, etc. The simulation conditions are:
- The ship makes sway movement as the sine curved shape around $\mathrm{x}, \mathrm{y}, \mathrm{z}$ axis. The periods of the oscillation around the three axes are $8 \mathrm{~s}, 7 \mathrm{~s}$, and $6 \mathrm{~s}$ respectively. The amplitudes of the oscillation are $4^{\circ}$, $5^{\circ}$, and $3^{\circ}$ respectively. The initial heading is $30^{\circ}$.

- The attitude angle increment output of LGU1 is the calculation result of the LGU. Output of LGU2 is adding the deformation angle increment data to the calculation result.

\section{B. Simulation results and analysis}

The method considering slow varying of static deformation needs to separate the static deformation and the dynamic deformation. The result is shown in Figure 3. Parameters of the models can be calculated accurately based on the separation result. Parameters of second-order Markov process are $\mu=[0.1,0.0625,0.0625](1 / \mathrm{s}), \lambda=[0.13,0.1,0.13]$ $(\mathrm{Hz}), \sigma=\left[9.8 \times 10^{-4}, 1.5 \times 10^{-3}, 4.7 \times 10^{-4}\right](\mathrm{deg})$. Parameters of zero-mean random walk process are $\delta=\left[3.7 \times 10^{-6}, 2.7 \times\right.$ $\left.10^{-6}, 7.3 \times 10^{-7}\right](\mathrm{deg})$
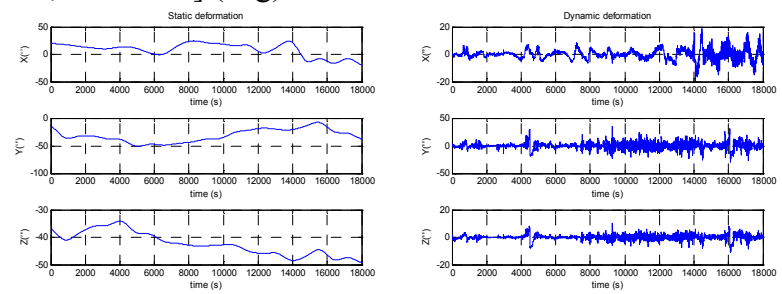

Figure 3. Static deformation and dynamic deformation.

Simulation results of $\Phi_{\mathrm{x}}$ are shown as follows. Figure.4 shows that mathematical models efficiently represented static and dynamic deformation separately. Add the two parts together and compare with the initial measurement data. Estimation result cannot be seen from figure. 5 because it is completely covered by the curve of the initial data. Estimation result almost is entirely consistent with the initial measurement data. Filtering out the high-frequency noise, the error curve is shown in figure.5. The STDs of the estimation error on three axes are $0.1086^{\prime \prime}, 0.1505^{\prime \prime}, 0.3112^{\prime \prime}$. To observe the simulation result visually, a part of $\Phi_{\mathrm{x}}$ is enlarged after filtering and the curve is shown in figure.6. The simulation results prove that this modeling method can represent the ship deformation with high accuracy.
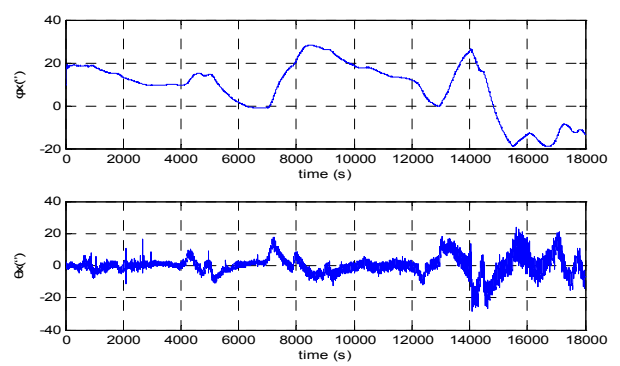

Figure 4. Simulation results of static and dynamic deformation. 

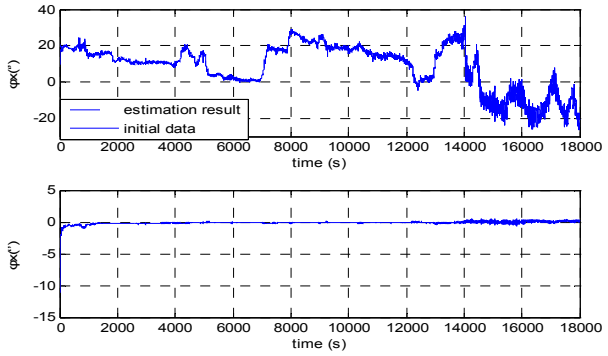

Figure 5. Simulation error.

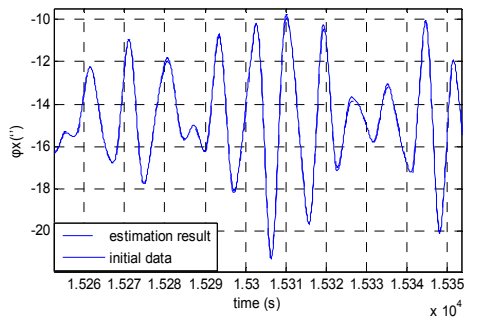

Figure 6. Comparison of simulation result and the initial measurement data in details.

Simulation results of the method identifying the static deformation to a constant value are shown in figure. 7 and figure.8. The static deformation and the initial deformation are not constant values in the simulation results and there is a clear trend in the simulation error curve. The reason is that the second-order Markov process cannot represent the ship deformation component in low frequency range. Due to the modeling method, the deformation component that cannot be represented couples to the static deformation and the initial deformation forcibly. So the ship deformation cannot be represented accurately by a single second-order Markov process. The deformation component in low frequency range needs to be represented separately.
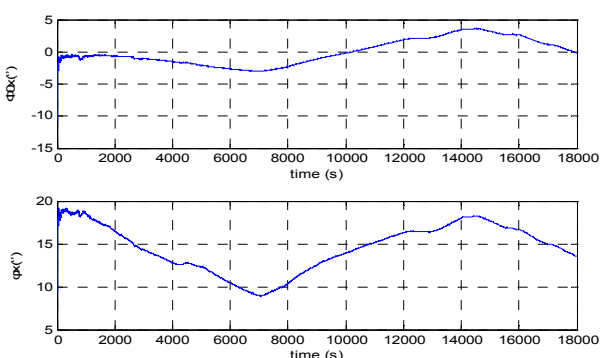

Figure 7. Simulation results of initial and static deformation.

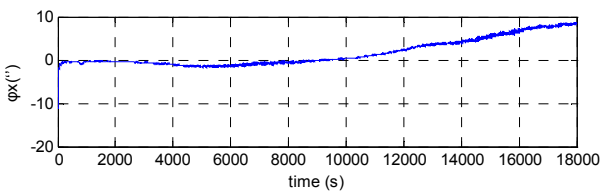

Figure 8. Simulation error.

\section{CONCLUTION}

This research proves that the real ship deformation can be represented accurately by two mathematical models together, a slow-varying variable and a second-order Markov process. The estimation precision can be better than 0.4" (STD). Parameters of the models can be calculated accurately by mathematical methods based on the wavelet analysis method. It ensures the high accuracy of the modeling. Second-order Markov process cannot represent the ship deformation component in low frequency range. So the method identifying the static deformation to a constant value is inaccurate. Based on the high-accuracy modeling method, the ship deformation can be measured accurately by inertial elements matching method after overcoming the errors brought in by the LGU system. The high-accuracy modeling method has a great value for engineering practice.

\section{ACKNOWLEDGMENT}

This research was supported by Science and Technology on Inertial Laboratory and Fundamental Science on Novel Inertial Instrument \& Navigation System Technology Laboratory.

\section{REFERENCES}

[1] A.V. Mochalov, A. Roumiantsev. Research of strapdown inertial navigation system on laser gyros in the attitude definition mode[C].Proceedings of SPIE, 2002.

[2] Mochalov A.V. A system for measuring deformation of large-sized objects[C]// RTO/ NATO. France, 1999, 15.1-15.9.

[3] Mochalov A.V,Kazantsev . Use of the ring laser units for measurement of the moving object deformation[C].Proceedings of SPIE, 2002(4680):85-92.

[4] I.M.Okon, I.B.Visegant, D.E Lukjanov. Three-ayas Laser Gyroscopes Used in Measurement Tasks of Relative Angular Position of Shipbome Systems[C]. Proceedings of the SecondSoviet-Cinese Symposium, Lenmgra, 1991:63 66.

[5] J.X. Zheng, S.Q.Qin, X.S.Wang. Attitude matching method for ship deformation measurement[J]. Journal of Chinese InertialTechnology,2010, 18(2): 175-180.

[6] Alan M. Schnider. Kalman Filter Formulations for Transfer Alignment of Strapdown Inertial Units [J].Navigation, 1983(30):72 89.

[7] Vaisgant I.B, Tupisev V.A, TyumenevaG.V. Sensitivity of Kalman filter in a task of definition of deformation of the ships deformation [J].Gyroscopy and Navigation, 1993(2):22 29.

[8] J.X. Zheng, S.Q.Qin, X.S.Wang. Ship hull angular deformation measurement taking slow-varying quasi-static component into account[J]. Journal of Chinese InertialTechnology,2010, 19(1): 6-10.

[9] M.Wen, H.Y.Ji. Spectrum denoise by wavelet analyze and its realize in Matlab[J]. Modern Electronics Technique, 2003,26(24):47-49. 